\title{
A Formal Model for Topic Maps
}

\author{
Pascal Auillans ${ }^{1}$, Patrice Ossona de Mendez ${ }^{2}$, \\ Pierre Rosenstiehl ${ }^{3}$, and Bernard Vatant ${ }^{4}$ \\ 1 LaBRI - Université Bordeaux 1, 351 cours de la Libération, Talence, France \\ auillans@labri.fr \\ 2 CNRS UMR 8557, 54 Bd Raspail, Paris, France \\ pom@ehess.fr \\ 3 CNRS UMR 8557, 54 Bd Raspail, Paris, France \\ pr@ehess.fr \\ 4 Mondeca, 3 cité Nollez, Paris, France \\ bernard.vatant@mondeca.com
}

\begin{abstract}
Topic maps have been developed in order to represent the structures of relationships between subjects, independently of resources documenting them, and to allow standard representation and interoperability of such structures. The ISO 13250 XTM specification 2] have provided a robust syntactic XML representation allowing processing and interchange of topic maps. But topic maps have so far suffered from a lack of formal description, or conceptual model. We propose here such a model, based on the mathematical notions of hypergraph and connexity. This model addresses the critical issue of topic map organization in semantic layers, and provides ways to check semantic consistency of topic maps. Moreover, it seems generic enough to be used as a foundation for other semantic standards, like RDF [3].
\end{abstract}

\section{Foreword: So Many Ways of Going Meta}

Meta-data, meta-level, meta-structure, meta-model, meta-language... going meta has been a popular game lately when it comes to create buzz words. Unfortunately the meta prefix has been used and abused in quite different meanings, in different contexts, application fields and communities. Those various applications now have to inter-operate in order to achieve Semantic Web consistent development.

Linguists, mathematicians and logicians all use the notion of meta-language, with maybe slight differences, but all pointing at the more abstract and generic level necessary to describe the structure and rules for a given language. Inside a language itself, every other assertion represents somehow a meta-level which organizes and structures the objects it makes assertions about. Naming and classifying make a meta-level for real world objects, while assertions make one for concepts, and more assertions and logical coordination for assertions.... The linguistic use of meta therefore implies a hierarchical semantic stratification.

In the Web universe, the proliferation of standards and specifications has led to the notion of meta-structure or meta-model. In XML, DTDs and Schemas 
have made familiar this notion in the form of standard document structure description.

But going meta does not mean necessarily going to an upper and more abstract structural level, it may mean going outside, after, before, besides or beyond, that is: in another semantic layer, to provide a description that is impossible to achieve from inside the reference layer. Illustration or annotation of a text document, and the other way round, caption of a figure, are basic examples. Generalizing annotation and caption, meta-data, providing standard description of resources, are considered a fundamental tool for Semantic web achievement.

\section{Are Topic Maps Meta-language or Meta-data?}

In this versatile meta-universe, where do topic maps stand? Some consider them as yet another meta-data format, based on the argument that the paradigm has explicitly split the world into two layers. The first layer contains subjects. Following topic maps terminology, a subject is whatever can be asserted about, be it abstract concept or real-world object, and is able to be represented by a topic in topic maps. The second layer contains addressable resources, which are whatever can be retrieved by computers in a network, singularly through URI addressing. The resources layer is the ground territory that subjects (topics) are mapping. Subjects, through their representation as topics, can be therefore considered as meta-data for resources. But topic maps represent also the structure of relationships between subjects, and therefore may be considered as a meta-language for structuring meta-data. In the "more meta than you" game, topic maps definitely rank well!

Considering that topic maps do not need any more organization in semantic layers,and that they could include their own meta-language, is therefore a tempting trap. The catchy slogan In topic maps, everything is a topic, has done nothing to prevent from this illusion of semantic homogeneity. And the implicit model under ISO 13250 XTM does not forbid whatever mismatch of semantic layers, leaving to human authors the entire responsibility of building in a consistent and meaningful way their structures of topics and associations. A formal model should make for this lack of rules, and provide means for checking consistency, through a formal definition of semantic layers. That is one of the main issues the present model will try to address.

\section{Introducing Hypergraphs}

The formal model we present here relies on the mathematical concept of hypergraph introduced by Berge in the Tihany colloquium in 1966 [7] (see also 6 6 810111824 ). Properties of hypergraphs and topological aspects (through hypermaps) have been extensively studied (see, for instance 9 12 14 19 23]).

This work extends the presentation done in the XML Europe 2001 conference [20]. 
Definition 1. A hypergraph is a 5-tuple $\mathcal{H}=\left(V, \lambda_{V}, E, \lambda_{E}, I\right)$, where:

- V,E,I are disjoint finite sets, which we call as follows:

- $V$ is the vertex set of $\mathcal{H}$,

- $E$ is the edge set of $\mathcal{H}$,

- $I$ is the incidence set of $\mathcal{H}$.

- $\lambda_{V}: V \rightarrow \mathcal{P}(I)$ is a mapping from $V$ to the set $\mathcal{P}(I)$ of all the subsets of $I$ (the image of a vertex is a subset of $I$ ), which we call the vertex-connector of $\mathcal{H}$, satisfying the following conditions:

$$
\begin{array}{r}
\forall v \neq v^{\prime} \in V, \quad \lambda_{V}(v) \cap \lambda_{V}\left(v^{\prime}\right)=\emptyset \\
\bigcup_{v \in V} \lambda_{V}(v)=I
\end{array}
$$

(notice that, for any incidence $\alpha \in I$, there exists thus a unique vertex $v \in V$, such that $\alpha \in \lambda_{V}(v)$, that we shall call the vertex-endpoint of $\alpha$ )

$-\lambda_{E}: E \rightarrow \mathcal{P}(I)$ is a mapping from $V$ to $\mathcal{P}(I)$, that similarly satisfies the following conditions:

$$
\begin{array}{r}
\forall e, e^{\prime} \in E, \quad \lambda_{E}(e) \cap \lambda_{E}\left(e^{\prime}\right)=\emptyset \\
\bigcup_{e \in E} \lambda_{E}(e)=I
\end{array}
$$

(as previously, for any incidence $\alpha \in I$, there exists a unique edge $e \in E$, such that $\alpha \in \lambda_{E}(e)$, that we shall call the edge-endpoint of $\alpha$ )

A vertex $x \in V$ and an edge $e \in E$ are incident if $\lambda_{V}(x)$ and $\lambda_{E}(e)$ have a non-empty intersection. By transitivity, we define the connected component $\mathcal{H}[x]$ of $x \in V \cup E$ : an element $y \in V \cup E$ belongs to $\mathcal{H}[x]$ if, and only if, either $y=x$ or there exists a (finite) sequence $z_{1}=x, z_{2}, \ldots, z_{k-1}, z_{k}=y$, such that $z_{i}$ is incident to $z_{i+1}(\forall 1 \leq i<k)$. Such a sequence is called a path from $x$ to $y$. We extend this definition to $V \cup E \cup I$ by setting $\mathcal{H}[\alpha](\alpha \in I)$ to be equal to $\mathcal{H}[x]$, where $x$ is the vertex-endpoint of $\alpha$.
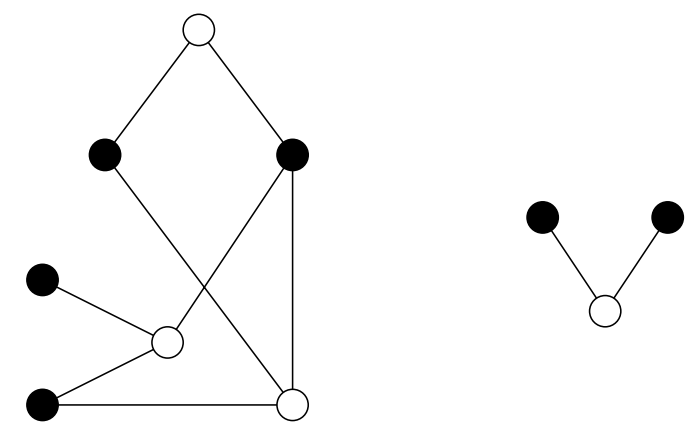

Fig. 1. A hypergraph (without multiple incidences), with two connected components 
In a first coarse interpretation, the hypergraph model seems to match only the main topic maps objects, called topic nodes and association nodes in the terminology of PMTM4 model [1]. Hypergraph vertices map to topic nodes, edges map to association nodes, and incidences map to links between topic nodes and association nodes. Every other topic map property, including role specification, scope, topic type, association template, topic occurrence, topic name, topic subject, implies in fact crossing boarders of semantic layers - represented by connected components. The topic map model has therefore to include a mechanism to jump between connected components. That mechanism is introduced in the following section.

\section{A General Model for Topic Maps}

Definition 2. A topic map is a triple $\mathcal{T}=\left(\mathcal{H}, \mathcal{H}^{\prime}, \phi\right)$, where:

- $\mathcal{H}=\left(T, \lambda_{T}, A, \lambda_{A}, I\right)$ is a hypergraph, the homogeneous hypergraph of $\mathcal{T}$, and we call

- topic set of $\mathcal{T}$, the vertex set $T$ of $\mathcal{H}$,

- association set of $\mathcal{T}$, the edge set $A$ of $\mathcal{H}$,

- incidence set of $\mathcal{T}$, the incidence set $I$ of $\mathcal{H}$.

$-\mathcal{H}^{\prime}=\left(X, \lambda_{X}, M, \lambda_{M}, I^{\prime}\right)$ is a hypergraph, the shift hypergraph of $\mathcal{T}$, and we call

- element set of $\mathcal{T}$, the vertex set $X$ of $\mathcal{H}^{\prime}$,

- meta-association set of $\mathcal{T}$, the edge set $M$ of $\mathcal{H}^{\prime}$,

- meta-incidence set of $\mathcal{T}$, the incidence set $I^{\prime}$ of $\mathcal{H}^{\prime}$.

- T, A, I, $M$ and $I^{\prime}$ are disjoint sets.

- The element set of $\mathcal{T}$ is the union of the topic set, the edge set and the incidence set of $\mathcal{T}$ :

$$
X=T \cup A \cup I
$$

- multiple incidences between a vertex and an edge of the shift hypergraph are not allowed:

$$
\forall(x, m) \in X \times M, \quad\left|\lambda_{X}(x) \cap \lambda_{M}(m)\right| \leq 1
$$

$-\phi: M \rightarrow T$ is an injective mapping (this means that two different elements are mapped to two different elements) from the meta-association set $M$ to the topic set $T$, that we call the lift of $\mathcal{T}$.

- For any meta-association $m \in M$ and any element $x \in X$, if $x$ is incident to $m$ in $\mathcal{H}^{\prime}$, then the lift $\phi(m)$ of $m$ does not belong to $\mathcal{H}[x]$ :

$$
\forall m \in M, \forall x \in X, \quad\left(\lambda_{X}(x) \cap \lambda_{M}(m) \neq \emptyset\right) \Longrightarrow(\phi(m) \notin \mathcal{H}[x])
$$




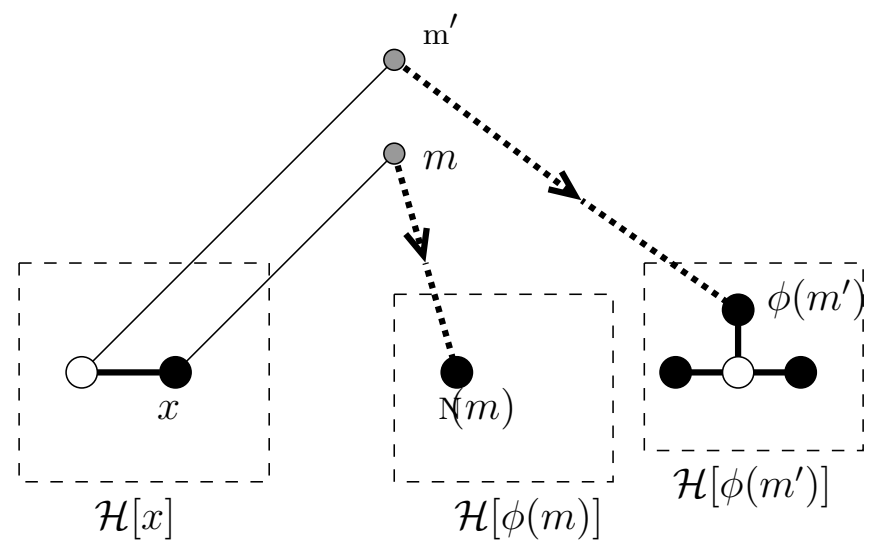

Fig. 2. A simple representation of the $\left(\mathcal{H}, \mathcal{H}^{\prime}, \phi\right)$ model

Property 7 justifies the terms of "homogeneous" and "shift" hypergraphs: intuitively, within a connected component of $\mathcal{H}$, all the topics, associations and incidences belong to the same semantic layer. Shifting of semantic layer is performed through a jump in the shift hypergraph.

\section{Example 1. (See Fig[3)}

The Solar System Topic Map 22] contains a certain number of semantic layers. In the connected component of $\mathcal{H}$ containing the "astronomical objects", a topic $j$ represents the planet Jupiter, of which vertex connector $\lambda_{T}(j)$ includes an incidence $\alpha$ representing the "satellite of the Sun" role, which in turn belongs to the edge connector $\lambda_{A}(a)$, where $a$ represents the "Sun Gravitational System" association. Those three elements belong to the same connected component of the homeogeneous hypergraph.

The topic $j$ (as an element of $X$ ) is incident, in $\mathcal{H}^{\prime}$, to a meta-association $m$, which corresponds (lifts) to "Solar System object" in a connected component of $\mathcal{H}$, representing another semantic layer (distinct from the one of $j$ ). The topics in this layer will represent classes of individual objects, and associations in this layer will be for instance class-subclass associations. The meta-incidence linking $j$ to $m$ thus represents the belonging of Jupiter to the topic type "Solar System object". Following the same principle, the association $a$ is incident in $\mathcal{H}^{\prime}$ to a meta-association (representing its relation to an association type) that lifts to "gravitational system". An identical mechanism lifts the incidences in the "objects" component of $\mathcal{H}$, into role types.

Example 2. It is possible to interpret (for part of the whole structure) the lift operation as a dual for the usual instantiation/derivation/representation of object oriented approaches. Such an interpretation may help to decompose a structure into semantic layers and give a strategy for building meta-associations and lifts, when the studied structure is somehow a "typing structure". We present figure 4 a possible structure for names and scopes. 


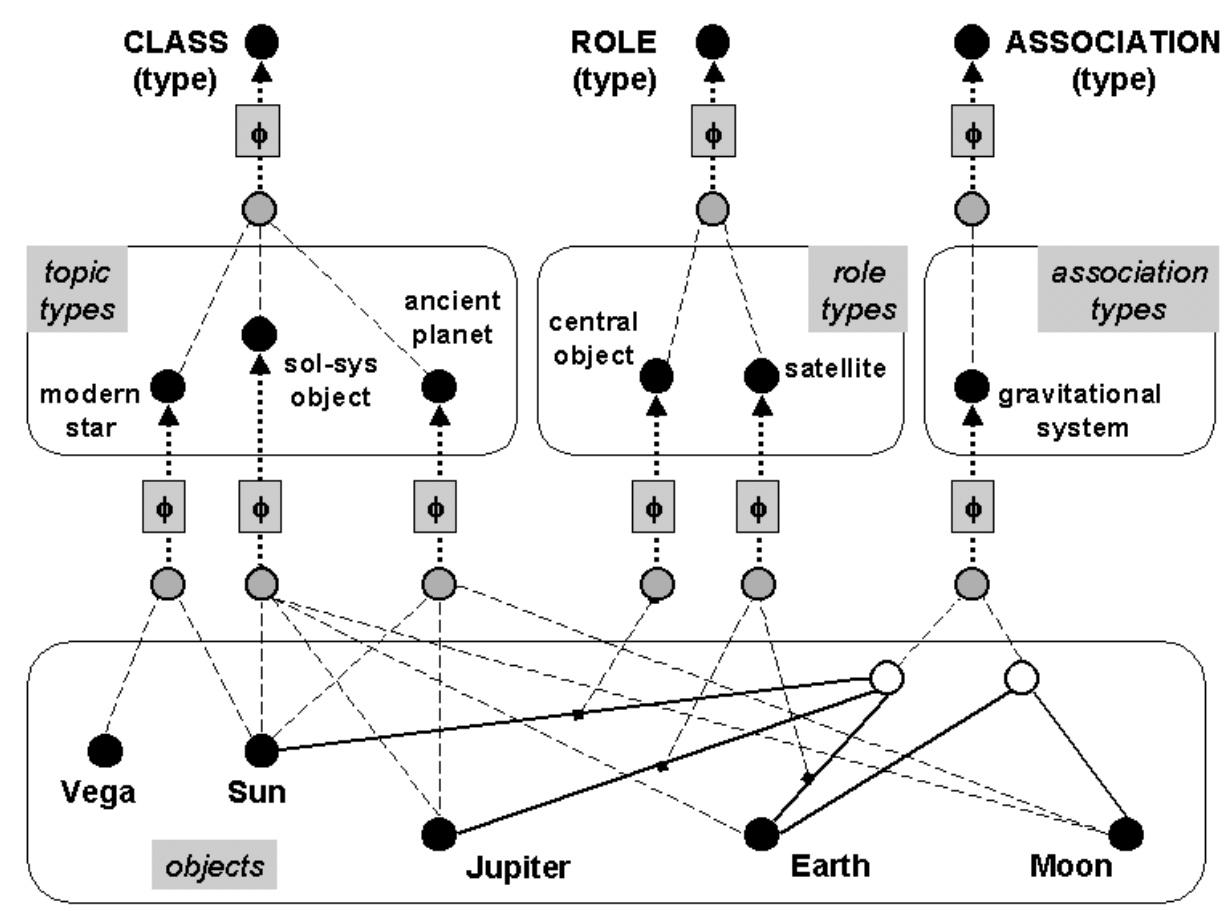

Fig. 3. Sample structure of a description of the solar system

\section{$5 \quad$ Lift and Fixed Point Properties}

In our model, Property (77) explicits that no connected component of the homogeneous graph may include a lift, which is consistent with the semantic of a lift, the change of semantic layer.

In practice, it is not always obvious to discover whether some topic characteristic should be coded through a lift, or if it should be coded using an association. In our model, a characteristic coded through a lift cannot have a fixed point. A consequence is that any characteristic having a fixed point has to be coded using an association.

Example 3. Consider, for instance, the data typing in a programming language like the C programming language. In such a language, types are only handled at compile time and there is a fundamental gap between types and data. Thus, it is possible to consider that the data and the types belong to two different semantic layers, and that the typing may be coded through a lift.

To the opposite, in a programming language like Pliant [21], types are objects, which in turn have a type. Obviously, the type Type is a fixed point for this relation. As the topic Type cannot belong to the same component as a topic obtained from it using exactly one lift, typing has to be coded by an association. Hence, all the types are connected to Type and, as all the data have to 


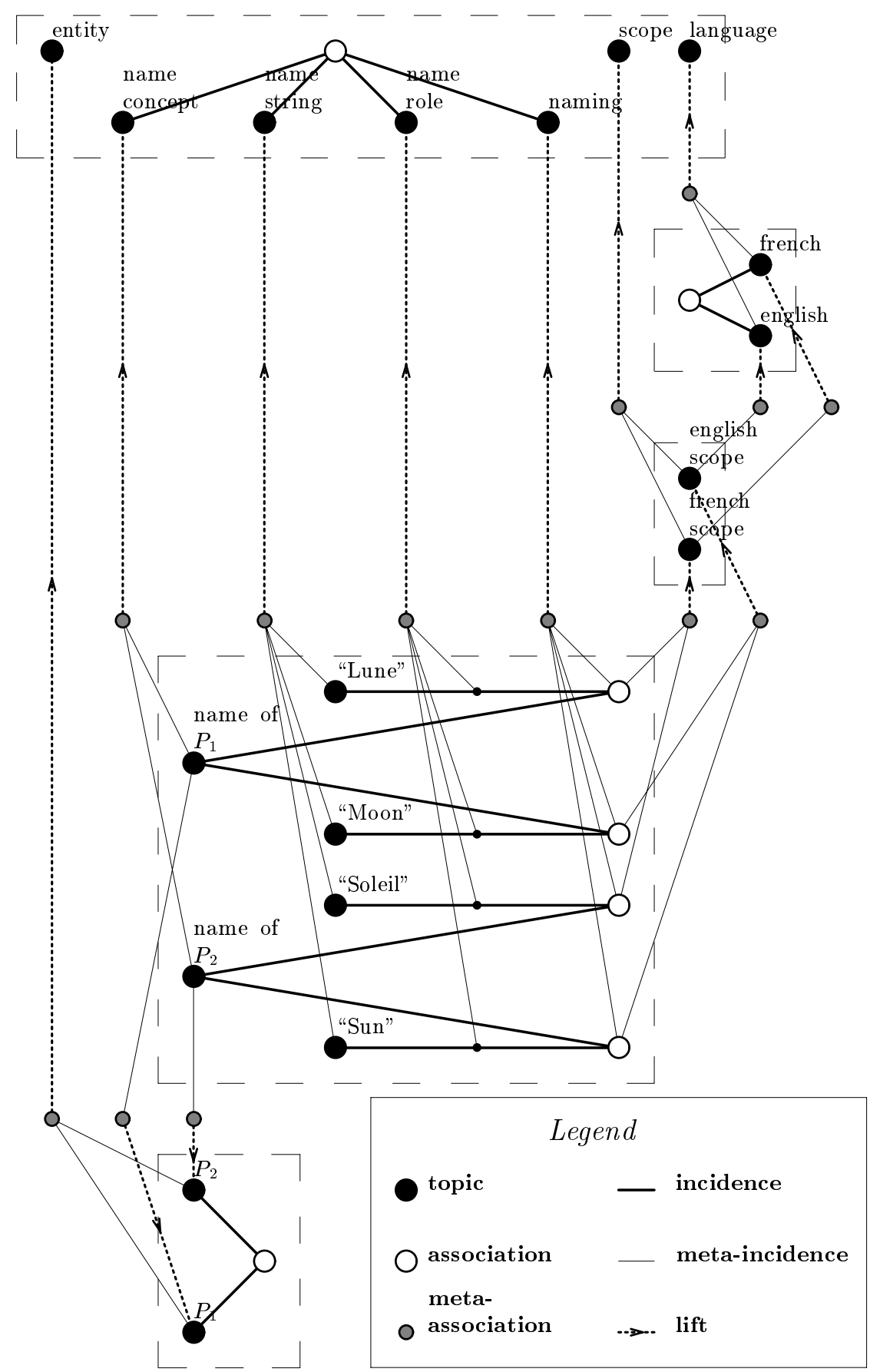

Fig. 4. Sample structure of names/scopes based on an interpretation of the lift dual to the instantiation/derivation/representation scheme . 
be connected to one type, all the data and types belong to a same connected component.

In topic maps used for knowledge representation, in order to keep semantic layers clearly distinct, it therefore seems relevant to avoid fixed points for fundamental characteristics such as topic type, association template, and role specification.

\section{Alternate $(\mathcal{H}, \theta)$ Description}

The previous considerations on semantic layers lead to an alternate description of the model, using only one hypergraph, and introducing another way to deal with connected components.

A topic map $\mathcal{T}$ may be defined as a couple $(\mathcal{H}, \theta)$, where:

$-\mathcal{H}=\left(T, \lambda_{T}, A, \lambda_{A}, I\right)$ is the homogeneous hypergraph of $\mathcal{T}$,

- $\theta: T \rightarrow \mathcal{P}(T \cup A \cup I)$ is a mapping from the topic set of $\mathcal{T}$ to the set of the subsets of elements of $\mathcal{T}$, that we call the covering of the topic map, with the constraint that

$$
\forall t \in T, \quad \mathcal{H}[t] \cap \theta(t)=\emptyset
$$

The equivalence of both descriptions is explicited now.
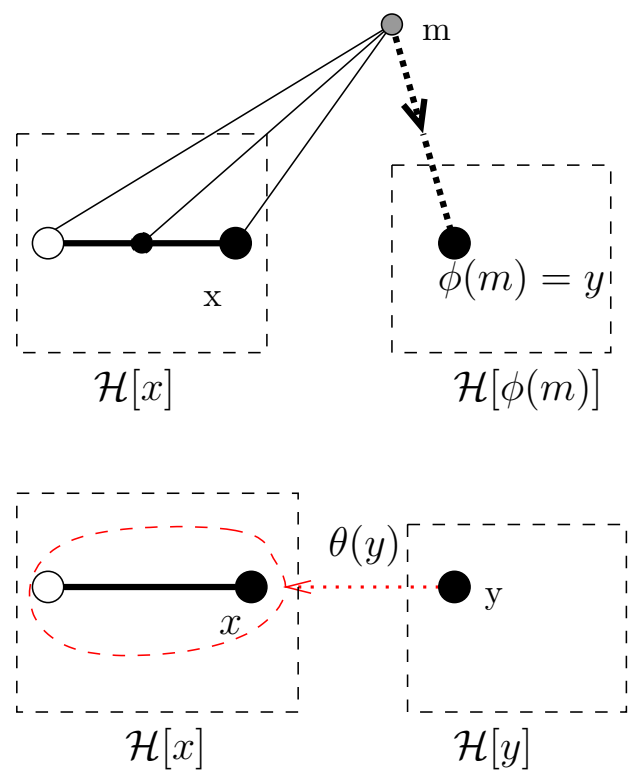

Fig. 5. Transformation of the $\left(\mathcal{H}, \mathcal{H}^{\prime}, \phi\right)$ model into the $(\mathcal{H}, \theta)$ one 
Consider a topic map. Let $\theta: T \rightarrow \mathcal{P}(T \cup A \cup I)$ be defined by:

$\forall t \in T, \quad \theta(t)= \begin{cases}\left\{x \in X, \lambda_{M}(m) \cap \lambda_{X}(x) \neq \emptyset\right\}, & \text { if } \exists m \in M, \phi(m)=t \\ \emptyset, & \text { otherwise }\end{cases}$

Then, (8) is a directed consequence of (7). Notice that $\theta$ is well defined by (9) as $\phi$ is injective.

Conversely, assume we are given a couple $(\mathcal{H}, \theta)$ satisfying (8). Let $M$ be a set of cardinality $\left|T \backslash \theta^{-1}(\emptyset)\right|$ and $\phi$ a bijection from $M$ to $T \backslash \theta^{-1}(\emptyset)$ (thus defining an injection from $M$ to $T$ ). Then, define:

$$
\begin{aligned}
X & =T \cup A \cup I \\
I^{\prime} & =\{(\alpha, t) \in X \times T, \quad \alpha \in \theta(t)\} \\
\lambda_{X}: x \in X & \mapsto\left\{(\alpha, t) \in I^{\prime}, \quad \alpha=x\right\} \subseteq I^{\prime} \\
\lambda_{M}: y \in M & \mapsto\left\{(\alpha, t) \in I^{\prime}, \quad \phi(m)=t\right\} \subseteq I^{\prime}
\end{aligned}
$$

then, denoting $\mathcal{H}^{\prime}=\left(X, \lambda_{X}, M, \lambda_{M}, I^{\prime}\right), \mathcal{H}^{\prime}$ is clearly a hypergraph and the triple $\left(\mathcal{H}, \mathcal{H}^{\prime}, \phi\right)$ is a topic map, as (7) is a direct consequence of (8).

Last, both transformations are obviously inverse from each other.

For example, in SolSysTM, the covering of "Solar System object" will contain the topics "sun", "moon", "earth", "Jupiter". The covering of "astrology" will contain the association "astrological interpretation of Moon-Jupiter occultation".

\section{Stratified Topic Maps}

We now introduce a subclass of topic maps, where the covering application induce a partial order over the set of connected components, and that we call stratified topic maps. This subclass seems fit to represent most practical use cases, and their model provide tools for checking semantic consistency of such topic maps, looking for loops and other semantic mismatches.

Given two connected components $\mathcal{H}[x]$ and $\mathcal{H}[y]$ of the homogeneous hypergraph of a topic map, we say that $\mathcal{H}[y]$ is $\theta$-reachable from $\mathcal{H}[x]$ and note $\mathcal{H}[x] \stackrel{\theta}{\rightarrow} \mathcal{H}[y]$ if there exists a sequence $\left(a_{1}, b_{1}, a_{2}, b_{2}, \ldots, a_{p}, b_{p}\right)$ (with $p \geq 1$ ), such that

$$
\begin{aligned}
b_{i} & \in \theta\left(a_{i}\right) & & (1 \leq i \leq p) \\
a_{i+1} & \in \mathcal{H}\left[b_{i}\right] & & (1 \leq i<p) \\
a_{1} & \in \mathcal{H}[x] & & \\
b_{p} & \in \mathcal{H}[y] & &
\end{aligned}
$$

A topic map is stratified if there exists no connected component $\mathcal{H}[x]$ of its homogeneous hypergraph, such that $\mathcal{H}[x] \stackrel{\theta}{\rightarrow} \mathcal{H}[x]$, which means that there are no "circuits" using lifts. 


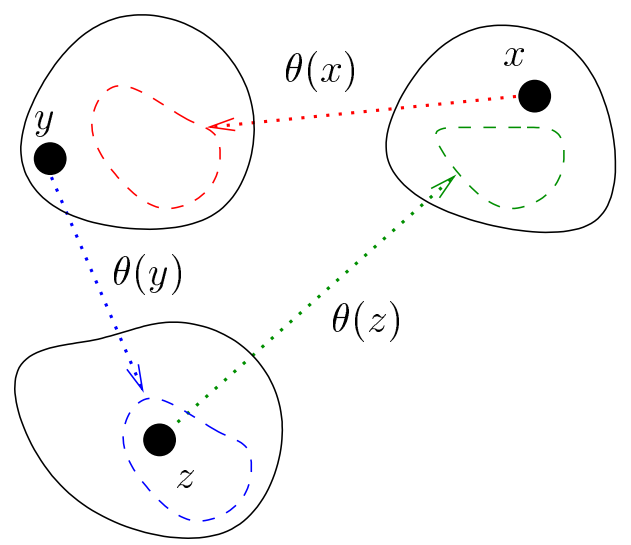

Fig. 6. A exemple of a non-startified topic map, since $\mathcal{H}[x] \stackrel{\theta}{\rightarrow} \mathcal{H}[x]$

In such a case, the set of all the connected components of the homogeneous hypergraph is partially ordered by

$$
\forall x, y \in X, \quad(\mathcal{H}[x]<\mathcal{H}[y]) \Longleftrightarrow(\mathcal{H}[y] \stackrel{\theta}{\rightarrow} \mathcal{H}[x])
$$

Considering a total order compatible with this partial order, the topic map may be unfolded and presented as a sequence $\left(\mathcal{H}_{1}, \ldots, \mathcal{H}_{p}\right)$ of disjoint (connected) hypergraphs with vertex set, edge set and incidence set $T_{i}, A_{i}$ and $I_{i}$, together with a set of mappings $\theta_{i, j}: T_{i} \rightarrow \mathcal{P}\left(T_{j} \cup A_{j} \cup I_{j}\right)$ (with $p \geq i>j \geq 1$ ). Condition (8) is thus replaced by the stronger assertion that $\theta_{i, j}$ mappings go from higher hypergraphs to a lower ones. The topic map may be conversely defined from the $\left(\mathcal{H}_{i}\right)$ sequence and the $\left\{\theta_{i, j}\right\}$ family, by:

$$
\begin{gathered}
\mathcal{H}=\bigcup_{i=1}^{p} \mathcal{H}_{i} \\
\theta: t \in T \mapsto \bigcup_{j=1}^{i-1} \theta_{i, j}(t) \subseteq T \cup A \cup I, \quad \text { where } i \text { is such that } t \in T_{i}
\end{gathered}
$$

\section{Structural Properties}

The practical study of the properties of a topic map will usually reduce to the study of the properties of components of its homogeneous hypergraph corresponding to a single semantic layer. Thus, we will be led to study structural properties of an hypergraph and will gain help from the extensive work already performed in this area. Notice that the power of hypergraph modeling for structure analysis is at the core of the use of such a model in domains like biochemistry to ease the study of probabilistic and topological systems with discrete interactions [1516. 
A fundamental type of relation between hypergraphs is the minor relation:

Definition 3 (from [10]). A hypergraph is a minor of another hypergraph $\mathcal{H}$, if it arises from $\mathcal{H}$ as the result of successive elementary operations, performed in any order. Elementary operations include the deletion of a vertex, of an edge, of an incidence ("shrinking"), and the identification of two vertices incident to a common edge ("collapsing").

This relation is the core of characterization theorems for classes of hypergraph, based on the following difficult theorem:

Theorem 1 (Robertson and Seymour 1987). Let $\mathcal{F}$ be a family of hypergraphs such that no hypergraph in $\mathcal{F}$ is isomorphic to a minor of another hypergraph in $\mathcal{F}$. Then $\mathcal{F}$ is finite.

As a consequence, if a class $\mathcal{C}$ of hypergraphs is stable by taking minors, there exists a finite set $\mathbb{F} \operatorname{orb}(\mathcal{C})$, such that, for any hypergraph $\mathcal{H}$, the hypergraph $\mathcal{H}$ belongs to $\mathcal{C}$ if and only if none of the hypergraphs in $\mathbb{F o r b}(\mathcal{C})$ is isomorphic to a minor of $\mathcal{H}$ (what may theoretically be tested in polynomial time for any fixed class $\mathcal{C})$.

\section{Organization Levels and Minors}

An important issue for our model stands in its ability to model a structure and a decomposition of it into organization levels.

In order to encode an organization level, we should keep in mind that any organization level has some "interface" with the underlying one:

"Whatever organization level we apprehend it, from the molecule level to the whole organization level, one now understands that an organic unit is an open system from the informational point of view, since each organization level receives its informations from the susjacent level." [17]

Let us now show that organization levels may be coded within our topic map model.

In such a context, organization levels are stratified, and each structure level is related to a layer hosting its messages and a layer hosting its functionalities. Considering a structure level $\mathcal{H}_{j}$, together with its message topics $\mathrm{MT}_{j}$ and its functionality topics $\mathrm{FT}_{j}$, it is natural to impose some semantic relations:

$$
\begin{aligned}
\forall t_{F} \in \mathrm{FT}_{j}, & \theta\left(t_{F}\right) \subseteq A \\
\forall t_{M} \in \mathrm{MT}_{j}, & \theta\left(t_{M}\right) \subseteq I \\
\forall t_{F}, t_{F}^{\prime} \in \mathrm{FT}_{j}, & \left(t_{F} \neq t_{F}^{\prime}\right) \Rightarrow\left(\theta\left(t_{F}\right) \cap \theta\left(t_{F}^{\prime}\right)=\emptyset\right) \\
\forall t_{M}, t_{M}^{\prime} \in \mathrm{MT}_{j}, & \left(t_{M} \neq t_{M}^{\prime}\right) \Rightarrow\left(\theta\left(t_{M}\right) \cap \theta\left(t_{M}^{\prime}\right)=\emptyset\right) \\
& A_{j}=\bigcup_{t_{F} \in \mathrm{FT}_{j}} \theta\left(t_{F}\right)
\end{aligned}
$$




$$
I_{j}=\bigcup_{t_{M} \in \mathrm{MT}_{j}} \theta\left(t_{M}\right)
$$

and an additional univocity relation:

$$
\forall t_{M} \in \mathrm{MT}_{j}, \forall a \in A, \forall t \in T, \quad\left|\theta\left(t_{M}\right) \cap \lambda_{A}(a) \cap \lambda_{T}(t)\right| \leq 1
$$

Then, if $j>i$, the structure level $\mathcal{H}_{j}$ will be a presentation of $\mathcal{H}_{i}$ at an highest organization level if the topics of the structure level $\mathcal{H}_{j}$ correspond to vertexdisjoint connected sub-hypergraphs of $\mathcal{H}_{i}$ and if the inter class incidences and associations of $\mathcal{H}_{i}$ correspond to the incidences and associations of $\mathcal{H}_{j}$, with a further correspondence with messages and functionalities in $\mathrm{MT}_{j}$ and $\mathrm{FT}_{j}$ :

Any element of $\mathcal{H}_{i}$ is either related to some topic in $T_{j}$ are to a message or to a functionality:

$$
\forall x \in T_{i} \cup A_{i} \cup I_{i}, \quad \exists ! y \in \mathrm{MT}_{j} \cup \mathrm{FT}_{j} \cup T_{j}, \quad x \in \theta(y)
$$

The associations and incidences of $\mathcal{H}_{i}$ belonging to the image by $\theta$ of $\mathrm{FT}_{j} \cup \mathrm{MT}_{j}$ are called inter-class associations and incidences

The image of a topic $t \in T_{j}$ in $\mathcal{H}_{i}$ has to be induced by the topic images:

$$
\begin{aligned}
& \forall a \in A_{i}, \quad(a \in \theta(t)) \Longleftrightarrow\left(\lambda_{A}(a) \subseteq \bigcup_{y \in \theta(t) \cap T_{i}} \lambda_{T}(y)\right) \\
& \forall \alpha \in I_{i}, \quad(\alpha \in \theta(t)) \Longleftrightarrow\left(\alpha \in \bigcup_{a \in \theta(t) \cap A_{i}} \lambda_{A}(a)\right)
\end{aligned}
$$

The functionality of any association of $\mathcal{H}_{j}$ is also the functionality of some corresponding inter-class association in $\mathcal{H}_{i}$ :

$$
\forall t_{F} \in \mathrm{FT}_{j}, \quad\left|\theta\left(t_{F}\right) \cap A_{i}\right|=1
$$

Incidences in $\mathcal{H}_{j}$ correspond to inter-class incidences in $\mathcal{H}_{j}$ related to a same message, that is: for any $t_{M} \in \mathrm{MT}_{j}$, for any $t_{F} \in \mathrm{FT}_{j}$, for any $t \in T_{j}$ and for any $a \in \theta\left(t_{F}\right) \cap A_{j}$, denoting $a^{\prime}$ the unique element of $\theta\left(t_{F}\right) \cap A_{i}$,

$$
\left(\lambda_{A}(a) \cap \lambda_{T}(t) \cap \theta\left(t_{M}\right) \neq \emptyset\right) \Longleftrightarrow\left(\exists t^{\prime} \in \theta(t), \lambda_{A}\left(a^{\prime}\right) \cap \lambda_{T}(t) \cap \theta\left(t_{M}\right) \neq \emptyset\right)
$$

Moreover, the image of a topic in $T_{j}$ has to induce a connected sub-hypergraph of $\mathcal{H}_{i}$, that is, denoting $N(X)$ (for any subset $X \subseteq T$ ) the union of $X$, of the set of the incidences with an endpoint in $X$ and of the set of the edges adjacent to a vertex in $X$ :

$$
\forall t \in T_{i}, \quad N\left(\theta(t) \cap T_{j}\right) \text { is connected }
$$


Actually, these relations implies that $\mathcal{H}_{j}$ is a minor of $\mathcal{H}_{i}$, what stresses the importance of the minor relation in the study of structural properties. One should also notice that our model naturally implies the existence of message and functionality topics, since any semantic shift correspond to a lift and thus to a topic. These topics are fundamentals: a message needs some material support to exist, some association needs some meaning to be pertinent.

\section{Conclusion}

Looking further on to applications, semantic consistency appears as a necessary condition for true interoperability. The founders of topic maps paradigm have constantly claimed their ambition to build a tool both allowing global and nonambiguous knowledge interoperability, and respecting semantic diversity. It is generally claimed that topic maps interoperability is ensured by non-ambiguous definition of subjects, their one-to-one representation by topics, and clear definition of core relationships through standard syntax. Diversity is ensured by the intelligent use of scope, allowing the coexistence of different or even contradictory assertions over the same set of topics.

The constraints introduced by the proposed model both set reasonable limits and conditions to such a program, and provide tools for its effective achievement, providing those conditions are respected. Through internal consistency checking, the model allows topic map builders and users to become aware of the actual semantic layers structure in their topic maps, clarify or modify their choices if necessary, fine-tune the structure and track semantic mismatches. On the other hand, by its very generic nature, the model does not impose any specific layering or semantics, and for that matter is consistent with the general topic maps principle of diversity.

The consequences of the model for merging and interoperability are stronger than they first could appear, and have to be stressed in this conclusion. As a matter of fact, the model allows distinction between syntactic merging and semantic merging. The syntactic merging seems the only one to have been addressed so far, in a view of topic maps where merging can always been achieved, whatever the origin of topic maps, provided correct syntax and process are used. But a very interesting consequence of our model is that internal semantic consistency of two separate topic maps does not guarantee the consistency of their merging. If two topic maps have different choices for organization of semantic layers, a relationship implemented here as an association might be implemented through a lift there. In such a case, the merging would either induce a semantic inconsistency, or need a correction to ensure consistency, in the merged map.

This new constraint on merging may appear at first sight as a serious drawback for the model, and a major obstacle to its interoperability. But in fact, it provides a way to check if any intended merging makes sense at all, therefore avoiding syntactically correct but meaningless merging. This leads to the notion of classes of topic maps supporting a common and consistent semantic layering. Such perspectives do not weaken the original paradigm, but on the contrary 
provide it with further accuracy and efficiency, and effective conditions of true interoperability.

\section{References}

1. PMTM4, a processing model for XML Topic Maps, http://www.topicmaps.net/pmtm4.htm, S. Newcomb and M. Biezunski (eds).

2. ISO/IEC 13250 topic maps, http://www.y12.doe.gov/sgml/sc34/document/0129.pdf, 1999, (since decembre 2001, the DTD of XTM 1.0 is included within the ISO 13250 specification).

3. Resource Description Framework (RDF) model and syntax specification, http://www.w3.org/TR/REC-rdf-syntax/, 1999, O. Lassila and R.R. Swick (eds).

4. Topic map foundational model requirements, http://www.y12.doe.gov/sgml/sc34/document/0266.htm, 2001, G. Moore and L.M. Garshol (eds).

5. XML Topic Maps (XTM) 1.0, http://www.topicmaps.org/xtm/1.0/, 2001, S. Pepper and G. Moore (eds).

6. C. Berge, Sur certains hypergraphes généralisant les graphes bipartis, Combinatorial Theory and its Applications (A. Rényi P. Erdös and V.T. Sös, eds.), North-Holland, 1970, pp. 119-133.

7. _ Graphes et hypergraphes, second ed., Dunod, Paris, 1973.

8. __ Hypergraphs, combinatorics of finite sets, North-Holland, 1989, (translation of Hypergraphes, Combinatoire des Ensembles Finis (Gauthier-Villars, Paris, 1987).

9. R. Cori and A. Machì, Maps, hypermaps and their automorphisms, Expo. Math. 10 (1992), 403-467.

10. P. Duchet, Hypergraphs, Handbook of Combinatorics (R.L. Graham, M. Grötschel, and L. Lovász, eds.), vol. 1, Elsevier, 1995, pp. 381-432.

11. P. Erdös and A. Hajnal, On chromatic number of graphs and set-systems, Acta Math. Acad. Sci. Hungar. 17 (1966), 61-99.

12. Z. Füredi, Matching and covers in hypergraphs, Graphs and Combinatorics 4 (1988), 115-206.

13. P. Hayes, RDF model theory, http://www.w3.org/TR/rdf-mt/, 2001.

14. D.S. Johnson and H.O. Pollak, Hypergraph planarity and the complexity of drawing Venn diagrams, Journal of Graph Theory 11 (1987), 309-325.

15. W. Klonowski, Probabilistic-topological theory of systems with discrete intercations. I. system representation by a hypergraph, Can. J. Phys. 66 (1989), 1051-1060.

16. _ Probabilistic-topological theory of systems with discrete intercations. II. calculation of the hypergraph probabilistic representation; the difference a posteriori algorithm, Can. J. Phys. 66 (1989), 1061-1067.

17. H. Laborit, La nouvelle grille, Laffont, 1974.

18. L. Lovász, Combinatorial problems and exercises, Akadémiai Kiadó/NorthHolland, 1979.

19. P. Ossona de Mendez, Representation of hypergraphs by contact of segments, Conférence Internationale "Graphe et Combinatoire", 1995.

20. __ Combinatorial hypermaps vs topic maps, XML Europe 2001, 2001, http://www.gca.org/papers/xmleurope2001/papers/html/s23-1.html.

21. H. Tonneau, The Pliant project, http://pliant.cx. 
22. B. Vatant, SolSysTM, a solar system topic map, http://www. universimmedia.com/topicmaps/solsystm.xml.

23. T.R.S. Walsh, Hypermaps versus bipartite maps, J. Combinatorial Theory 18(B) (1975), 155-163.

24. A.A. Zykov, Hypergraphs, Uspeki Mat. Nauk 6 (1974), 89-154. 